\title{
Anti-VEGF Anticalin PRS-050-PEG40
}

National Cancer Institute

\section{Source}

National Cancer Institute. Anti-VEGF Anticalin PRS-050-PEG40. NCI Thesaurus. Code C91376.

A pegylated, proprietary lipocalin that targ ets human vascular endothelial growth factor (VEGF), with potential antineoplastic activity. Pegylated anti-VEGF anticalin PRS-050 specifically targ ets and binds to VEGF receptor 2 (VEGFR2 or KDR), thereby preventing its activity. This may inhibit angiogenesis and eventually reduce tumor cell growth. 